\title{
THE DIFFERENTIAL DIAGNOSIS OF RHEUMATIC FEVER
}

\author{
BY \\ K. A. HALLIDIE-SMITH and E. G. L. BYWATERS \\ From the Rheumatism Research Unit (Medical Research Council), Canadian Red Cross Memorial Hospital, Taplow
}

(RECEIVED FOR PUBLICATION JANUARY 29, 1958)

Since the efficacy of continuous daily sulphonamide or penicillin administration in the prevention of attacks of rheumatic fever has now been established, and the measure recommended not only nationally but by international committees (World Health Organization, 1957), it has become increasingly important to recognize even mild cases of rheumatic fever. At the same time it is equally essential to avoid diagnosing this erroneously, since the resulting continuous daily prophylaxis is undesirable from the point of view both of the patient and of the tax-payer. We thought therefore that an account of our observations at this National Centre for Juvenile Rheumatism over the last nine years would be useful, since over this period there has accumulated considerable experience not only of rheumatic fever, but also of many conditions which masquerade as such.

Out of such prolonged experience at the House of the Good Samaritan in Boston, Duckett Jones (1944) was able to formulate a set of diagnostic criteria which have proved extremely useful in a modified form (Rheumatic Fever Committee Joint Report, 1955) for ensuring in a large therapeutic trial that cases from many different centres were of the same general type. He realized that cases of other diseases were as far as possible to be ruled out. It was realized further that many cases of genuine rheumatic fever, if first observed late in the course of the disease, or if mild, could not be fitted in and would have to be called 'probable rheumatic fever'. Furthermore, some of the criteria depend upon continuous clinical observation or laboratory tests usually possible in hospital, but only comparatively rarely made in this country in the average general practice. In this centre then, a discharge diagnosis of rheumatic fever has been made on unformulated but rather wider grounds than those of the Duckett Jones criteria, so that of 2,214 patients admitted to the unit over the nine-year period, 959 were discharged with the diagnosis of rheumatic fever. Nearly two thirds of these satisfied diagnostic criteria comparable to those of Duckett
Jones, save that chorea was excluded as a major criterion and past rheumatic fever as a minor criterion. In addition, 42 cases were discharged with a diagnosis of 'possible rheumatic fever' and 202 with a diagnosis of Still's disease, the remaining 1,011 cases being various other miscellaneous disorders.

\section{Group A. Erroneous Initial Diagnoses in Rheumatic Fever Cases}

A one-in-six sample of these patients ultimately diagnosed as rheumatic fever was taken, consisting of those with surnames beginning with the initials A, B and C admitted between July, 1947, and June, 1956. In this group of 160 rheumatic fever patients, 12 were found definitely to have been initially otherwise diagnosed, though subsequently all satisfied Duckett Jones' criteria for the diagnosis of rheumatic fever. All were children between the ages of 6 and 12 years, apart from the patient initially thought to have sub-acute bacterial endocarditis, and only in that patient and the child thought to be a case of Still's disease was the ultimate diagnosis not made before transfer here. All patients have been regularly followed up since discharge, for periods between 18 months and seven years.

Poliomyelitis. These two children complained of fever and limb pains and one had neck stiffness. Normal cerebrospinal fluid was found and in both significant heart murmurs became audible subsequently.

Pneumonia. This 9-year-old girl gave a history of general malaise and limb pains. On admission to the referring hospital, she was febrile and was found to have impaired percussion note and crepitations in the right mid-zone. Following subsidence of the chest signs she developed significant heart murmurs, and later rheumatic nodules.

Meningitis. This child had a sore throat followed by limb pain and headache. Normal cerebrospinal fluid was obtained and she subsequently developed polyarthritis and heart block.

Rubella. This 7-year-old girl had a sudden onset of abdominal pain and a rubella-like rash. There was, how- 
ever, a normal white count and no accompanying lymphadenopathy; she subsequently developed pericarditis and endocarditis.

Dysentery. This girl complained of abdominal pain, diarrhoea and limb pains. Stool culture was negative. She complained of limb pains after the diarrhoea settled and an organic mitral pansystolic murmur developed.

Subacute Bacterial Endocarditis. This young adult female with known rheumatic heart disease gave a five-day history of general malaise, generalized aches and pains and fever. She subsequently developed polyarthritis and a raised antistreptolysin- 0 titre; repeated blood cultures were negative and there were no embolic manifestations. She responded to bed rest and salicylates.

Appendicitis. These two children both complained of abdominal pain. Normal appendices were removed, but they both subsequently developed polyarthritis and organic heart murmurs.

Suppurative Arthritis of Hip. This 9-year-old boy had a four weeks' history of pain in one hip with inguinal adenitis and fever. He subsequently developed polyarthritis and a mitral pansystolic murmur.

Still's Disease. This boy presented with a three months' history of polyarthritis, fever, rash and general malaise. It was not until after 10 weeks in this hospital that he developed heart lesions, by which time the arthritis had settled leaving no residua.

Fallen Arches. This 6-year-old boy had complained of pain in his feet for four weeks and had been treated with remedial exercises. He developed polyarthritis, and had a raised E.S.R. and A.S.O. titre.

\section{Group B. Unsubstantiated Admission Diagnosis of Rheumatic Fever}

This group consisted of all patients admitted between July, 1947, and October 31, 1956, with the presumed diagnosis of rheumatic fever, but in whom this diagnosis was not substantiated. Only those were included in whom there was fairly certain evidence from the referring letter or history on entry that they were thought to have rheumatic fever; they numbered 196.

These 196 admissions consisted of 171 children under the age of 17 years, and 25 adults. Follow-up has varied from none in a small minority to several years in some instances. There has been no evident rheumatic activity at these check-ups, and no patient has developed organic heart disease under outpatient observation. None of the patients either on long or short term follow-up has been re-admitted with rheumatic fever. They may be divided into seven broad groups which can be further subdivided as shown in Table 1. The various groups will now be considered in more detail:
TABLE 1

FINAL DIAGNOSIS OF 196 CASES REFERRED AS RHEUMATIC FEVER IN WHOM THIS DIAGNOSIS WAS NOT CONFIRMED

Functional (8):

Physiological tachycardia

Hysteria . . . .

Traumatic and Postural (11):

Sprains $\ldots$.

back ache-1 each ...

Infections (91)

Upper respiratory tract

Lower respiratory tract

Bone and joint

Blood struam

Uninary t:act

Pericarditis

Specific fevers

Connective Tissue Disorders (42):

Still's and rheumatoid arthritis

Schonlein-Henoch purpura

Erythema nodosum

Visceral lupus erythematosus

Periarteritis nodosum

Penicillin sensitivity

Granuloma annulare

Rheumatic and Congenitai Heart Disease (9):

Congenital

Rheumatic

Blood Dyscrasias (2):

Iron deficiency anaemia

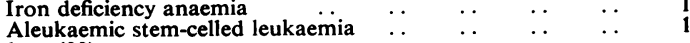

Others (33):

Arthritis.

Arthralgia

Limb pains

Fever and syphilis

Total

I. Functional. This group comprised six children and two young adults. The three patients with physiological tachycardia were all children. The first had a history of recent sore throat and the referring doctor thought he had detected clinical signs of cardiac enlargement with persistent tachycardia. The second child had complained of limb pains for six weeks and was noted to have an evening temperature up to $98 \cdot 8^{\circ} \mathrm{F}$. and persistent tachycardia of 130-140 per minute. The third child had a recent history of cold and cough with persistent tachycardia. Nothing abnormal could be found in any of these patients, and, although the pulse rate was elevated by day, the sleeping pulse was within the normal range (Nelson, 1954).

The two young female adults complained of limb pains and general ill-health; one had had rheumatic fever as a child; she showed hysterical manifestations such as hyperventilation and no organic signs were found. The other patient also had nothing organic demonstrable, and was referred to a psychiatrist who diagnosed neurosis and depression.

The other three children all complained of pains in joints or limbs for which no organic cause was found. One had a functional wrist drop, the second hysterical gait, and the third, with an unhappy home background, admitted to exaggerating his symptoms 
because of desire for sympathy. All were helped by psychiatric treatment.

II. Traumatic and Postural. All the patients in this group were children, and all complained of joint pains; three patients had a history of rheumatic fever. In eight patients there was a definite history of trauma with objective signs in a single joint (knee in three cases, ankle in two, hip in two and elbow in one) with radiological evidence of slipped epiphysis in the two patients with hip involvement and of osteochondritis dissecans in the patient with elbow involvement. In the other five patients, there were normal serological findings, normal hearts and absence of other joint involvement. One patient gave a history of rheumatic fever previously, and of recent joint pains, low grade fever and suspected presystolic murmur; she was found to have a normal heart and normal temperature range, but a considerable degree of sternal deformity and scoliosis. Another girl had also had rheumatic fever and complained of pains in her back and thighs. No signs of rheumatic activity were found, but the backache was thought to be postural following her period of prolonged bedrest. The last patient gave a history of painful swelling of both knees following a sore throat. She had a normal heart and there was no evidence of rheumatic activity. Both knees tended to lock in flexion and to extend suddenly with a click, suggesting meniscus lesions. Exploration of the knee joints revealed torn cartilages, which were thought to be due to a congenital defect.

Slipped Femoral Epiphysis. (i) This girl, aged 13, was referred with the following letter from her family doctor, 'Rheumatic fever 18 months ago. Now complains of pain in joints, right foot swollen. Systolic murmur at apex which has just appeared. I should be grateful if you would take her in for recurrence of rheumatic fever'. The history obtained on transfer here was of trauma to the right thigh one month previously, since when she had limped and noticed pain in the feet, knee and hip on the affected side, with recent swelling of the foot. On examination, movements of the right hip were restricted and painful and there was slight swelling of the right foot. A radiograph of the right hip showed slipped femoral epiphysis. There was evidence of mitral and aortic valve disease but this was thought to be inactive and she was transferred to an orthopaedic hospital for operation. She has now been followed up for five years and there has been no fresh rheumatic activity.

(ii) J.H., aged 14, was admitted with painful swelling of the elbow for two weeks. He had had rheumatic fever with carditis two years previously. After admission no further joints became involved and there was no evidence of recent streptococcal infection or carditis; but radiography showed osteochondritis dissecans affecting the capitellum.
III. Infections. (a) UPPER ResPiRATORY TRACT INFECTIONS. Of this large group, 45 (43 children and two young adults) were diagnosed as upper respiratory tract infections. The presumptive diagnosis of rheumatic fever was based on preceding upper respiratory tract infection followed by limb or joint pains, fever and general malaise in 33 patients. There were 12 patients in the whole group who did not complain of pain and in eight of them an organic systolic murmur was initially suspected, as in 16 of those who did complain of limb and joint pains. Three had had previous rheumatic fever. In all these patients, rheumatic activity was thought unlikely because of the absence of abnormal physical signs other than those of a present upper respiratory tract infection in some cases, and because of normal or rapidly settling sedimentation rate and negative evidence of streptococcal infection, except for two children (one with raised A.S.T.) whose throat swabs grew group A haemolytic streptococci.

(b) LOWER Respiratory TRACT INFECTIONS. All these patients were children and the typical symptomatology differed from the upper respiratory tract group in that fever and cough were the presenting symptoms; pain, complained of by four children, was confined to chest or shoulder. Two children (one with primary tuberculosis and the other with bronchiectasis) had persistently elevated sedimentation rates and in four patients an organic systolic murmur was queried but not confirmed here. There was radiological proof of diagnosis in six cases (two, primary tuberculosis; one, bronchiectasis and three, lobar pneumonia). The remaining four children were diagnosed as bronchitis or resolving pneumonia in view of the chest signs on admission and the absence of cardiac involvement or streptococcal infection.

Left Lower Lobe Pneumonia. This girl, aged 8, had had rheumatic fever and chorea five years previously. She had a sore throat one week before present admission followed by general malaise and fever. Her doctor queried precordial friction rub and thought she had had a relapse of acute rheumatism. This was not confirmed here but there were early signs of pneumonia at the left base and a subsequent radiograph showed consolidation of the left lower lobe. She responded to penicillin therapy.

(c) Bone AND JoInt InfeCtions. Four of these 10 patients (nine children and a young adult male) complained of multiple joint pains and fever, but subjective and objective signs eventually became localised to one joint and diagnosis was confirmed by obtaining pus on drilling or by periosteal changes on radiographic examination. The six other patients had pain and swelling of a single joint with fever, and diagnostic proof by aspiration of purulent fluid in five cases and radiological changes in three (periostitis in two and Brodie's abscess in the third). 
J.W., aged 15, was admitted to another hospital with fever and pain in the right knee. White blood cells were 8,000 per c.mm. with $80 \%$ polymorphs. Osteomyelitis was queried and she was given a short course of penicillin, but she then developed pain in other joints and was thought to have rheumatic fever. She was put on to salicylates and transferred here. She continued to run a low grade pyrexia and high E.S.R. and a bony swelling of the lower end of the right femur became apparent. White blood cells at this time were 5,000 per c.mm. with $50 \%$ polymorphs. Radiographs showed marked bony changes of osteomyelitis and the femur was subsequently drilled.

(d) Blood Stream Infections. Three of these cases were children and the fourth a young adult female. All complained of multiple acute painful swollen joints, fever and general malaise. In one boy an organic mitral systolic murmur was suspected. Ultimate diagnosis was based on a positive blood culture (staphylococci.in two cases and streptococci in two cases), rapid response to chemotherapy and absence of cardiac involvement.

(e) URinary Tract Infections. These four patients (all children) complained of limb pains and general malaise and were referred with low grade fever and a suspected organic systolic murmur in one case. All had Esch. coli urinary infections and one had a hydronephrosis. No other abnormal physical signs were found, and there was no evidence of carditis or of streptococcal infection.

$(f)$ Pericarditis. One of these children presented with shoulder pain and fever, and the referring doctor thought that organic systolic and diastolic murmurs were present. On admission here she was found to have pericardial and pleural effusions with pericardial friction, but no evidence of endocarditis. There was no evidence of streptococcal infection. A Mantoux test at 1:1,000 was positive and radiographs showed enlarged hilar glands on one side. Symptoms and signs resolved after prolonged bed rest, and follow-up for two years showed satisfactory progress. The other child complained of general malaise and chest pain and his general practitioner found him to be febrile and thought that an organic systolic murmur was present. On admission, he had a pericardial effusion and friction rub, but no evidence of endocarditis. There was no evidence of streptococcal infection, but a Mantoux test at $1: 10,000$ was positive. In both these cases, the tentative diagnosis on discharge was tuberculous pericarditis, but in view of their satisfactory progress in hospital and at follow-up, in retrospect they may both have been cases of benign non-specific pericarditis.

(g) SPecific Fevers. This group comprised 13 children and three girls aged 18-19 years. Five children had an acute episode of fever, limb pains and general malaise. This fever settled quickly after admission, no abnormal physical signs were elicited and there was no abnormal serology. They were thought to be cases of influenza, though no serological proof of diagnosis was sought.

Four patients had a discharge diagnosis of glandular fever. They all had fever and limb pains and in two of them an organic systolic murmur was questioned by the referring doctor. They were diagnosed after development of generalized lymphadenopathy and splenomegaly, abnormal mononuclear leucocytes in the peripheral blood and a positive Paul Bunnell titre in one case. No abnormal cardiac signs were noted and findings reverted to normal quite quickly.

An example of this group was a girl, aged 10. She was admitted with a history of sore throat three weeks previously and five days' fever and joint pains. On admission here she had a fever of $99^{\circ} \mathrm{F}$. but no abnormal physical signs. Erythrocyte sedimentation rate was $15 \mathrm{~mm}$./hr. and a white cell count was normal. Five days after admission, a white cell count showed a relative lymphocytosis of $60 \%$ and a week later $23 \%$ abnormal mononuclears were noted in a total of 15,850 W.B.C. per c.mm. Paul Bunnell titre was positive (1:224).

Six patients had acute histories with fever and muscle and joint pains in each case, but rapidly changing physical signs, finally developing features typical of measles, poliomyelitis, meningitis (no organism isolated but response to chloramphenicol therapy), infective hepatitis, erysipelas and paratyphoid fever.

The paratyphoid fever patient, an 18-year-old girl, had had rheumatic fever previously, leaving established mitral and aortic valve lesions. She gave a three-day history of fever, headache, generalized aches and pains with vomiting and diarrhoea for two days. The doctor thought she had had fresh rheumatic activity and put her on to salicylates. On admission here, vomiting and diarrhoea was a noticeable feature, and Paratyphosus $B$ was isolated from blood, urine and faeces. She responded to treatment with chloramphenicol.

The patient with poliomyelitis, aged 4 years, was referred with the following letter from the general practitioner: 'History of tonsillitis two days previously. On admission she developed severe rheumatic pains in the arms and shoulders. Refuses to move the right arm because of the pain. C.N.S., all reflexes present and equal.' On admission here she was found to be febrile with slight neck stiffness and a flaccid paralysis of the right arm. The cerebrospinal fluid showed changes consistent with poliomyelitis.

The last patient in this group, a 13-year-old boy, had an acute onset of high fever, pain and weakness in one hip. There was no evidence of cardiac involvement nor of streptococcal infection and no further joints became involved; it was thought that he might have had radiculitis of virus origin. 
IV. Connective Tissue Disorders. (a) Still's Disease AND Rheumatoid ARthritis. These 24 cases (three of whom were over 16 years of age) all presented with joint pains and the majority had had objective joint signs prior to admission; fever and general malaise were also presenting features. Eighteen had had acute symptoms and signs for over a month prior to admission here. Persistence of 'active' joints, swinging fever and in some cases lymphadenopathy or typical Still's rash, with confirmation by biopsy of synovial membrane in less classical cases, furnished the eventual diagnosis. Of these patients, five had pericarditis and in three patients a significant systolic murmur was thought to be present by the referring doctor. This latter finding was not confirmed here. It is of interest to note that while 16 of the 18 with symptoms of over one month's duration were thought to be Still's disease on entry to this hospital, all those six with histories of under one month's duration were thought to be rheumatic fever when first admitted; underlining the considerable difficulty in the early recognition of Still's disease. The following case history illustrates this point:

J.F., aged 11. Nine days before admission here he developed pains in his thighs after playing football. Next day the left ankle and right toe became swollen and painful. The general practitioner found his E.S.R. to be $72 \mathrm{~mm}$./hr. and put him on salicylates. On admission here the right big toe and left ankle were hot and swollen, and the right hip painful. E.S.R. was $60 \mathrm{~mm}$./hr.; heart and electrocardiogram normal. He was thought to be a case of rheumatic fever and put on cortisone. Despite high dosage, acute arthritis persisted and the left elbow also became involved. Persistent joint involvement with soft tissue thickening and raised E.S.R. with no evidence of endocarditis, favoured the alternative diagnosis of Still's disease which has been confirmed by the subsequent progress of the patient.

(b) Schonlein-Henoch Purpura. Of the eight cases with Schonlein-Henoch purpura, five had polyarthritis and three had limb pains; abdominal pain was a prominent feature in four cases. In six patients there was history of preceding upper respiratory tract infection. In all cases the diagnosis was eventually clarified by the appearance of typical petechiae.

(c) Erythema Nodosum. There were two adults and three children in this group. Four patients had joint pains and two had joint swelling together with fever. All developed lesions typical of erythema nodosum and the illness settled spontaneously, without an obvious cause having been discovered. Radiographs of the chest were normal and throat and nose swabs negative with no rise in antistreptolysin-O titre. The fifth patient gave a history of severe sore throat and fever treated with sulphathiazole, and he subsequently developed lesions typical of erythema nodosum. Sulphathiazole was suggested as the provocative agent after exclusion of tuberculosis.

( $g$ ) Generalized Lupus Erythematosus. Two young females were eventually diagnosed as acute lupus erythematosus. These patients both had joint pains and pericarditis. Eventual diagnosis was made in the first patient by the development of typical skin lesions with biopsy proof and L.E. cells found in peripheral blood. In the second patient proof was eventually found at autopsy. In neither was the diagnosis obvious at the time of transfer.

(e) Periarteritis Nodosa. In this child of 11 , a sore throat was followed two weeks later by polyarthritis, fever and rash of multiform type. On transfer here she had a patchy reticular erythema, pericarditis, polyarthritis and muscle pains. Skin biopsy showed changes typical of periarteritis nodosa. Subsequent follow-up has confirmed this diagnosis.

( $f$ ) Probable Penicillin Sensitivity. This 5-yearold boy presented with pains in his knees and swelling of his face following an upper respiratory tract infection treated with penicillin. There was no evidence of arthritis, carditis or streptococcal infection on transfer, and he was thought to be sensitive to penicillin.

(g) Granuloma AnNulare. This patient, a 6-yearold boy, gave a history of nodules on elbows, wrists, toes and neck, and pain in his knees, for six months. On admission here he had a normal E.S.R. and antistreptolysin-O titre. Nodule biopsy resembled granuloma annulaire, though there is no record of his subsequent progress.

V. Rheumatic and Congenital Heart Disease. (a) Congenital Heart Disease. These two children were referred on account of general malaise and the discovery of abnormal heart murmurs. One had signs typical of coarctation of the aorta, and the other, aged 4, was admitted with a history of a severe febrile cold following which a heart murmur had been noted. On admission here there was no evidence of rheumatic activity, but cardiovascular findings were those of patent ductus arteriosus, which was subsequently confirmed at operation two years later.

(b) Rheumatic Heart Disease. This group comprised six children and a 19-year-old girl, all of whom had had rheumatic fever previously with established rheumatic heart disease. The recent history was of joint or limb pains, general malaise and tachycardia. All had normal sedimentation rate, antistrepto- 
lysin-O titre and sleeping pulse, and there was no evidence of active rheumatism.

VI. Blood Dyscrasias. (a) Iron DeficienCy ANAemia. This 14-year-old girl presented with general malaise and odd aches and pains; the referring doctor thought there was a significant heart murmur. She was found to have severe iron deficiency anaemia and an innocent basal systolic murmur. Despite full investigation, no satisfactory explanation was found other than inadequate diet.

(b) Aleukaemic Stem-Celled Leukaemia. This 6-year-old girl was admitted from another hospital with a diagnosis of rheumatic fever. There was one month's history of general malaise with joint pains, fever, tachycardia and swelling of the left knee. E.S.R. was $32 \mathrm{~mm} . / \mathrm{hr}$.; Hb., $13 \cdot 8 \%$; W.B.C., $7,000 / \mathrm{mm}^{3}$ (polymorphs, $82 \%$, leucocytes, $16 \%$, monocytes, $2 \%$ ). There was an unsatisfactory response to salicylates. She was thought here to be a case of rheumatic fever until, following progressive weight loss, mild generalized lymphadenopathy and anaemia, the bone marrow was examined and showed a picture of aleukaemic stem-celled leukaemia. Radiographs of the long bones showed mild generalized osteoporosis with coarse mottling.

VII. Others. This group consisted of 25 children and eight adults. In only one patient, an adult male, aged 42, could a definite diagnosis be made. He had polyarthritis and a basal diastolic murmur. These findings were confirmed here. He had a raised serum uric acid and radiological changes characteristic of gout; the Wassermann reaction was positive, accounting for the aortic incompetence. In the other 32 patients, the provisional diagnosis of rheumatic fever was not confirmed but no satisfactory alternative diagnosis could be found. Follow-up has varied from none, in a small minority, to seven years, and in no case have rheumatic fever-like episodes recurred, nor has any cardiac abnormality developed. They are grouped according to their main symptomatology as follows:

(a) Arthritis of UneXPlained Origin. These patients, four children and one young female adult, gave a history of joint swellings but had no abnormal physical signs on admission here. No evidence of streptococcal infection was found (normal antistreptolysin-O titre and negative nose and throat swabs) and normal E.S.R. Short term follow-up was satisfactory.

(b) Arthralgia. These patients gave a history of joint pains dating over several weeks or months and when examined by the family doctor there was either a slight rise in temperature, tachycardia or a suspected organic murmur. Three patients gave a history of recent upper respiratory tract infection. Clinical and serological examinations were negative in all these patients. Short term follow-up was satisfactory.

(c) Limb PaIN. These patients all complained of limb pains, four following an upper respiratory tract infection. In four cases the referring doctor queried an organic systolic murmur; another patient had had rheumatic fever previously, and in another there was a strong family history of rheumatic fever. In other patients, low grade fever and/or tachycardia were mentioned in the referring letter. When admitted here, all investigations were negative.

(d) FEVER. These patients were referred on account of unexplained febrile illnesses during which the general practitioner thought that an organic murmur was present. This finding was not confirmed here, and the cause of the preceding febrile episode was never established.

\section{Observations}

From the above analysis, it may be seen that many diagnoses may be confused with that of rheumatic fever. In Group B on closer study, four general categories of diagnostic errors became evident: 1. Those cases who would fulfil the Duckett Jones criteria but in whom further observation and development of fresh features provided the ultimate correct diagnosis. 2 . Those who would have fulfilled the criteria if all points in the history and clinical examination could have been corroborated. 3 . Those in whom there were never even questionably sufficient criteria. 4 . Those in whom the diagnosis might have been rejected or the correct one established if the referring doctor had had the facilities and leisure for carrying out further investigations.

The majority in the first category are found in the connective tissue diseases, notably rheumatoid arthritis. The early acute stage of this disease closely resembles rheumatic fever, especially in children, with pericarditis, salicylate-responding pyrexia and transient joint involvement; the Rose Waaler test is usually negative. Only continued observation of the subsequent course of the disease, or the development of endocarditis, erythema marginatum or chorea provides a solution. Even chorea may be seen in acute lupus erythematosus.

The second category comprised both those in whom the referring doctor's findings were not substantiated here, or were not felt to be significant in the diagnosis of rheumatic fever, and also cases in which other points were elicited that had either been missed before or developed subsequently. In greater detail these notably concern the following: 
(1) History. A careful history is an essential in the exclusion or confirmation of the less typical forms of acute rheumatism. In several instances the relationship to trauma was missed; in a large number it seemed that complaints of limb or joint pains contributed too prominently towards the proposed initial diagnosis of rheumatic fever, since they were not complained of on transfer here and did not recur. A previous history of rheumatic fever or a family history of the illness often seemed to swing the balance disproportionately in favour of a presumptive diagnosis of rheumatic fever.

(2) Fever. Temperatures of $98 \cdot 8^{\circ}-99 \cdot 4^{\circ} \mathrm{F}$. were often cited as being a significant feature. As an isolated finding in a child, this is probably of little consequence, since a normal child's temperature may often reach these levels at some time in the 24 hours.

(3) Tachycardia. This was a feature often stressed in referring letters, particularly in the group which presented primarily with limb and joint pains. Tachycardia out of proportion to the temperature has not been a common finding here, usually occurring only in those children with severe rheumatic fever or cardiac failure; in a few cases of acute rheumatism there has been a sinus bradycardia in the early stages. Even mild apprehension can cause tachycardia in a nervous child, but the sleeping pulse will be within normal range for the age group.

(4) Systolic Murmur. The presence of an organic systolic murmur was initially queried in a total of 48 patients, half of whom were in the upper respiratory tract infection group. A systolic murmur can be demonstrated in a high percentage of infants (Hallidie-Smith, unpublished work) and most normal children (Lessof and Brigden, 1957). These murmurs are usually best heard down the left sternal edge or just internal to the apex. They may be fairly localized or propagated out of the apex and up to the neck. The innocent systolic murmur never fills systole (Lessof and Brigden, 1957), and is usually maximal in early or mid systole. The character may be low pitched and musical, or rather more high pitched and blowing (Friedman, Robie and Harris, 1949). The precise aetiology of these murmurs has not been established, but they are thought to be related to flow of blood in the great vessels and are accentuated in the febrile child whose cardiac output is increased. In contrast, the organic systolic murmur is smooth and high-pitched, filling systole. It is best heard at, or just internal to, the apex, and is propagated outwards to the axilla.

The last category comprises those in whom the error of diagnosis might not have been made if the referring doctor had been able to carry out further investigations toward an alternative diagnosis, since there was often insufficient evidence for suggesting the diagnosis as it stood. The sedimentation rate is of particular value. A normal rate (less than $15 \mathrm{~mm}$./hr. Westergren) at the onset of the disease, would make the diagnosis of active acute rheumatism very unlikely, although it may remain above this level for as little as two weeks. A search for evidence of a preceding streptococcal infection by isolation of the organism in throat or nose swab, or demonstration of a rise in antistreptolysin-O titre, is of some help in doubtful cases.

In other published series (McCue and Galvin, 1948; Lewy, 1952; McCue, 1954; Saslaw, Hernandez and Werblow, 1954; Wedum and Rhodes, 1955) similar points have been made about history-taking, examination and evaluation of physical signs; in each, a large proportion of cases were referred with a provisional diagnosis of rheumatic fever, which either could not be substantiated or was superseded by an alternative diagnosis, sometimes only after careful observation over a period of time. The good long-term prognosis noted in the group with upper respiratory tract infection followed by fever and limb pains is confirmed by these writers and by Kaiser (1943). In the series referred to, the overall pattern of differential diagnosis approximates to our own with certain exceptions, in part explained by the different geographical distributions of disease, e.g. sickle cell anaemia, and in part by the fact that some series only dealt with out-patient referrals. Amongst other less common diseases not listed in our series, but noted in the literature with reference to the differential diagnosis of rheumatic fever, are cat scratch fever (Lyon, 1956); meningococcaemia (Daniels, 1948); miliary tuberculosis (Freud, Weisz and Brunhofer, 1950); ascariasis (Doumer, Lorriaux and Belbenoit, 1951); juvenile hyperparathyroidism (Bogdonoff, Woods, White and Engel, 1956); Weber Christian panniculitis (Brudno, 1950); Hodgkin's disease (Paquet and Delage, 1957); berylliosis (Sprague and Hardy, 1954), sarcoidosis (Myers, Gottlieb, Mattman, Eckley and Chason, 1952); hepatitis (Martini, 1950), and irritable hip syndrome (Caravias, 1956). This last interesting entity has also been noted here. The symptoms are fever, general malaise and sudden onset of severe pain confined to the hip, all settling spontaneously and thought to be due to synovitis.

\section{Summary}

The differential diagnosis of rheumatic fever is discussed from the experience at Taplow over a nine-year-period from 1947 to 1956. The original diagnosis is reviewed in a sample of those cases 
ultimately diagnosed as rheumatic fever, and 12 cases are described which were originally diagnosed otherwise.

A larger series of 196 patients is described who were originally thought to have rheumatic fever, but in whom this diagnosis was eventually not substantiated. This series is subdivided into seven main diagnostic disease groups, which are discussed in detail and illustrated. The pointers in clinical investigation that led to the eventual diagnosis are listed and common erroneous interpretations of history, fever, tachycardia and systolic murmurs are discussed.

Thus the differential diagnosis of rheumatic fever still may present considerable difficulties, particularly in its milder forms. Some of these difficulties are insurmountable. Others could be overcome by more rigid adherence to diagnostic criteria, by more critical examination of the problems presenting, and by increased awareness of the significance of the physical signs elicited. Fuller investigations at an early stage in the disease, notably sedimentation rate and a search for streptococcal infection, would be helpful in the less obvious cases.

We are indebted to Dr. Wallace Brigden, Dr. Reginald
Lightwood, Dr. Gerald Thomas and Dr. Paul Wood for their guidance and participation over the years.

\section{REFERENCES}

Bogdonoff, M. D., Woods, A. H., White, J. E., and Engel, F. L. (1956). Amer. J. Med., 21, 583

Brudno, J. C. (1950). New Engl. J. Med., 243, 513

Caravias, D. E. (1956). Arch. Dis. Childh., 31, 415

Daniels, W. B. (1948). Arch. intern. Med., 81, 145.

Doumer, E., Lorriaux, A. and Belbenoit, C. (1951). Bull. Soc. méd. Hôp. Paris, 67, 801 .

Freud, P., Weisz, A. and Brunhofer, A. (1950). Amer. J. Dis. Child., 79,676

Friedman, S., Robie, W. A. and Harris, T. N. (1949). Pediatrics, 4, 782 .

Hallidie-Smith, K. A. Unpublished work.

Jones, T. Duckett (1944). J. Amer. med. Ass., 126, 481

Kaiser, A. D. (1943). N.Y. St.J. Med., 43, 1937

Lessof, M. and Brigden, W. (1957). Lancet, 2, 673

Lewy, F. J. (1952). Ann. intern. Med., 36, 1042.

Lyon, R. (1956). Lancet, 2, 555.

McCue, C. M. (1954). J. Pediat., 44, 290.

- and Galvin, L. F. (1948). Ibid., 33, 467

Martini, G. A. (1950). Dtsch. med. Wschr., 75, 1464.

Myers, G. B., Gottlieb, A. M., Mattman, P. E., Eckley, G. M. and

Chason, J. L. (1952). Amer. J. Med., 12, 161.
Nelson, W. E. (1954). Textbook of Pediatrics, 6th ed., p. 854. Saunders, Philadelphia.

Paquet, E. and Delage, J. M. (1957). Canad. med. Ass. J., 76, 927.

Rheumatic Fever Committee Joint Report (1955). Rheumatic Fever Working Party of the Medical Research Council of Great Britain and the Subcommittee of Principal Investigators of the American Council on Rheumatic Fever and Congenital Heart American Council on Rheumatic Fever and Congenital Heart

Saslaw, M. S., Hernandez, F. A. and Werblow, S. C. (1954). J. Pediat., 44, 414.

Sprague, H. B. and Hardy, H. L. (1954). Circulation (N.Y.), 10, 129. Wedum, B. G. and Rhodes, P. H. (1955). J. Amer. med. Ass., 157, 981.

World Health Organization. (1957). Report of the Committee on Rheumatic Diseases. 